\title{
Low-Complexity User Scheduling with Switched Tilting for 3D Cellular Systems
}

\author{
Haijing Liu, Hui Gao, and Tiejun Lv \\ School of Information and Communication Engineering, Beijing University of Posts and Telecommunications, Beijing 100876, China \\ Correspondence should be addressed to Tiejun Lv; lvtiejun@bupt.edu.cn
}

Received 6 October 2014; Revised 28 February 2015; Accepted 4 March 2015

Academic Editor: Hsuan-Ling Kao

Copyright (C) 2015 Haijing Liu et al. This is an open access article distributed under the Creative Commons Attribution License, which permits unrestricted use, distribution, and reproduction in any medium, provided the original work is properly cited.

\begin{abstract}
We propose a low-complexity user scheduling scheme to enhance the sum rate performance for a multicell downlink system, in which the base station (BS) is equipped with a large-scale active antenna array. First, we divide each cell into $N$ regions according to the vertical beamwidth of the BS antennas. Next, candidate user equipment (UE) items are assigned to corresponding groups to their locations. Each scheduling slot is also divided into $N$ equal-time subslots. Then, at each subslot, we focus on one UE group, select the optimal number, $K^{*}$, of UEs for simultaneous data transmission in the manner of round-robin scheduling, and adjust the BS antenna tilting to the optimal angle $\theta_{\text {tilt }}^{*}$. In particular, $K^{*}$ and $\theta_{\text {tilt }}^{*}$ for each UE group are both obtained by means of largesystem asymptotic analysis. Benefiting from the random matrix theory tools, the asymptotic analytical results are independent of instantaneous channel state information of UE, which make it possible to solve $K^{*}$ and $\theta_{\text {tilt }}^{*}$ offline, therefore saving the online computational resources significantly. Numerical results verify that the proposed scheme achieves good sum rate performance with extremely low computational complexity.
\end{abstract}

\section{Introduction}

In order to satisfy the exponentially increasing demand for mobile data traffic, many new technologies, such as millimeter wave, large-scale antenna systems (LSAS) [1] and active antenna systems (AAS) [2], are emerging in wireless communications. AAS are capable of offering significant signal enhancement and intercell interference reduction through electronic beam control in both horizontal and vertical domains [3, 4]. LSAS have attracted considerable attention for achieving huge spectral-efficiency and energy-efficiency gains. However, most of the existing LSAS works discuss the systems with omnidirectional antennas (see [1] and references therein). The large-scale AAS (LS-AAS) have not been fully studied so far. Only [5] introduces the 3D cell planning with LS-AAS.

As one of the most important factors of large-scale fading (LSF) in AAS, the antenna tilting design is critical to system performance. The dynamic adaptation of vertical antenna tilting for AAS has become a hot topic, and many valuable researches emerge [6-11]. However, the conventional antenna tilting optimizations $[6,7]$ are usually user-specific and wellperformed when the number of served user equipment (UE) items is small. When it comes to LSAS with a large number of UE items, an enormous amount of online computation would be required for the antenna tilting optimization. The switched tilting schemes in $[3,8]$ could avoid the high-complexity optimization of the user-specific tilting methods in $[6,7]$, but we cannot apply them to LS-AAS directly. To be more specific, some important factors (e.g., the optimal number of active UE items [12]), which play critical roles in LSAS while not being too important in common MIMO systems, have not been considered carefully in the existing switched tilting schemes. Moreover, though [5] discusses the 3D planning of LSAS cells, it focuses on evaluating the sum rate performance of cooperative minimum mean square error (MMSE) receivers. Effective UE scheduling with antenna tilting adjustment has not been considered.

In this paper, we propose a low-complexity user scheduling scheme together with switched BS antenna titling, aiming to improve the sum rate performance of a multicell downlink system. The BSs are equipped with large-scale active 


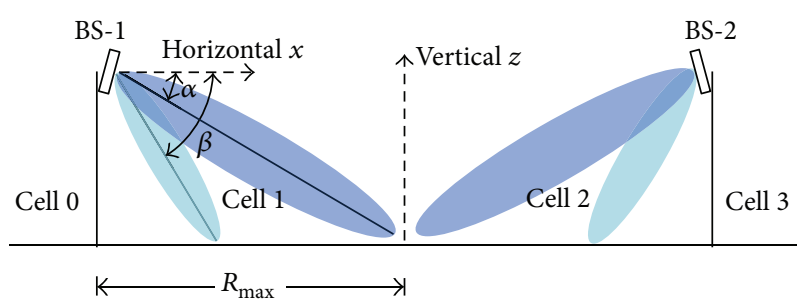

FIGURE 1: Multicell system model with various BS downtilts.

antenna arrays and perform zero-forcing ( $\mathrm{ZF}$ ) precoding. In the proposed scheme, UEs are divided into $N$ groups and accordingly a scheduling slot consists of the same number of equal-time subslots. At the $n$th subslot $(n=1, \ldots, N)$, the $\mathrm{BS}$ adjusts the antenna tilting angle to the optimal value $\theta_{n}^{*}$ and schedules the optimal number $K_{\text {tilt, } n}^{*}$ of UE items in the corresponding UE group for simultaneous data transmission in each cell. To be more specific, the main contributions of our work are as follows. (i) Considering the vertical beam width of the BS antennas, we design an effective UE partition approach, by which the UEs are assigned to groups according to their locations. (ii) Leveraging random matrix theory(RMT-) based large-system analyses, we obtain the approximate sum rate of each UE group, which is independent of the instantaneous channel state information (CSI) of UE. The optimal number of active UE items, $K_{n}^{*}$, and the optimal value of antenna tilting angle, $\theta_{\mathrm{tilt}, n}^{*}$, for each UE group are then jointly solved offline to maximize the average approximate sum rate. (iii) During each subslot, we adopt the round-robin scheduling (RRS) method to select UE; neither instantaneous CSI nor online computation is required for the scheduling, making the proposed scheme much more applicable in practice.

Notations. We use uppercase boldface letters for matrices and lowercase boldface for vectors. $(\cdot)^{H},(\cdot)^{\dagger}, \operatorname{tr}(\cdot)$, and $\mathrm{E}[\cdot]$ denote the conjugate transpose, the pseudoinverse, the trace, and the expectation, respectively. $\mathscr{C} \mathscr{N}(\mathbf{m}, \boldsymbol{\Theta})$ denotes the circularly symmetric complex Gaussian distribution with mean vector $\mathbf{m}$ and covariance matrix $\Theta$. $\stackrel{\text { a.s. }}{\longrightarrow}$ denotes almost sure convergence.

\section{System Model}

We consider a multicell MIMO downlink system, in which each cell consists of a BS and a large number of single-antenna candidate UE items. The BS is with a large-scale active antenna array. The number of BS antennas is denoted by $M$, and the total number of candidate UE items is denoted by $C$. As shown in Figure 1, the one-dimensional Wyner cellular model [15] is adopted. In other words, we assume all the BSs are located on a straight line and the UE items in a cell are only interfered by the nearest two adjacent BSs. We focus on the performance of Cell 1 and treat Cell 0, Cell 2 as the interfering cells.

A scheduling slot is divided into $N$ equal-time subslots, and the BSs utilize RRS method to select UE items. At the $n$th $(n=1, \ldots, N)$ subslot, $K_{n} \mathrm{UE}$ (called active UE) is scheduled for simultaneous data transmission in Cell 1. The received signal $\mathbf{y}_{n} \in \mathbb{C}^{K_{n} \times 1}$ at UE in Cell 1 is given by

$$
\mathbf{y}_{n}=\mathbf{G}_{n} \mathbf{x}_{n}+\mathbf{G}_{n}^{\prime} \mathbf{x}_{n}^{\prime}+\mathbf{G}_{n}^{\prime \prime} \mathbf{x}_{n}^{\prime \prime}+\mathbf{n}_{n}
$$

where $\mathbf{G}_{n} \in \mathbb{C}^{K_{n} \times M}, \mathbf{G}_{n}^{\prime} \in \mathbb{C}^{K_{n} \times M}$, and $\mathbf{G}_{n}^{\prime \prime} \in \mathbb{C}^{K_{n} \times M}$ denote the channel matrices from BS-1, BS-0, and BS-2 to the scheduled UE in Cell 1 , respectively. $\mathbf{x}_{n} \in \mathbb{C}^{M \times 1}$ is the postprecoded signal transmitted from BS-1 to the UE in Cell 1. $\mathbf{x}_{n}^{\prime} \in \mathbb{C}^{M \times 1}$ and $\mathbf{x}_{n}^{\prime \prime} \in \mathbb{C}^{M \times 1}$ are the signal vectors transmitted from BS-0 and BS-2 to UE in Cell 0 and Cell 2, respectively. $\mathbf{n}_{n} \in \mathbb{C}^{K_{n} \times 1}$ with i.i.d. $\mathscr{C} \mathscr{N}\left(0, \sigma_{n}^{2}\right)$ entries is the AWGN vector at the UE. To simplify notation, hereafter we will drop the subscript $n$ for indexing subslot. The channel matrix $\mathbf{G}$ is given by (the assumptions of our CSI model, including the Wyner model, the large number $M$ of BS antennas, and the simplified antenna pattern, relieve the system performance analysis from complicated derivations. We adopt them for the proof of concepts. In fact, they might not be quite feasible in practice (e.g., currently, it is hard to deploy a huge array consisting of 64 active antenna elements at a transmitting site). If we remove these assumptions, the system model would become more apposite to practical systems, but the performance analysis would be very complicated.)

$$
\mathbf{G}=\mathbf{D H}
$$

where $\mathbf{D} \in \mathbb{R}^{K \times K}$ is a diagonal matrix indicating the LSF CSI from BS-1 to the UE in Cell $1 . \mathbf{H} \in \mathbb{C}^{K \times M}$ with i.i.d. $\mathscr{C} \mathscr{N}(0,1)$ entries is the small-scale fading (SSF) CSI matrix. The $k$ th diagonal element $\sqrt{d_{k}}$ of $\mathbf{D}$ is modeled as

$$
\sqrt{d_{k}}=\sqrt{l_{k}^{-\alpha} 10^{A_{k} / 10}}
$$

where $l_{k}$ is the distance from the $k$ th UE to the BS- 1 antennas, $\alpha$ is the path-loss exponential factor, and $A_{k}$ in $\mathrm{dB}$ is the BS-1 antenna gain to the $k$ th UE. In this paper, we adopt the BS antenna radiation pattern proposed by $3 \mathrm{GPP}$ in [13]; that is,

$$
\begin{gathered}
A_{H}(\varphi)=\min \left(12\left(\frac{\varphi}{\varphi_{3 \mathrm{~dB}}}\right)^{2}, A_{\mathrm{max}}\right), \\
A_{V}(\theta)=\min \left(12\left(\frac{\theta-\theta_{\text {tilt }}}{\theta_{3 \mathrm{~dB}}}\right)^{2}, \mathrm{SLL}_{v}\right), \\
A(\varphi, \theta)=-\min \left(A_{H}(\varphi)+A_{V}(\theta), A_{\max }\right),
\end{gathered}
$$

in which $\varphi$ denotes the horizontal angel between the BS antenna array boresight and the UE, $\theta$ is the vertical angle between the horizon and the UE location, and $\theta_{\text {tilt }}$ is the downtilting angle of the BS antenna. $A_{\max }$ represents the maximum antenna attenuation, and $\mathrm{SSL}_{v}$ is the tilt side lobe level in the vertical plane of the BS antenna pattern. $\varphi_{3 \mathrm{~dB}}$ and $\theta_{3 \mathrm{~dB}}$ stand for the half-power beamwidth (HPBW) in the horizontal and vertical plane, respectively. Like [16], we simplify the analysis by assuming $A_{\max }=\mathrm{SSL}_{v}=\infty, A(\varphi)=0$. Hence, $d_{k}$ can be rewritten as

$$
d_{k}=l_{k}^{-\alpha} 10^{-1.2\left(\theta_{k}-\theta_{\mathrm{tilt}}\right)^{2} / \theta_{3 \mathrm{~dB}}^{2}} .
$$


Similarly, the interfering channel matrices $\mathbf{G}^{\prime}$ and $\mathbf{G}^{\prime \prime}$ are given by $\mathbf{G}^{\prime}=\mathbf{D}^{\prime} \mathbf{H}^{\prime}$ and $\mathbf{G}^{\prime \prime}=\mathbf{D}^{\prime \prime} \mathbf{H}^{\prime \prime}$, in which $\mathbf{D}^{\prime}=$ $\operatorname{diag}\left(\sqrt{d_{1}^{\prime}}, \ldots, \sqrt{d_{K}^{\prime}}\right)$ and $\mathbf{D}^{\prime \prime}=\operatorname{diag}\left(\sqrt{d_{1}^{\prime \prime}}, \ldots, \sqrt{d_{K}^{\prime \prime}}\right)$, respectively. $d_{k}^{\prime}$ and $d_{k}^{\prime \prime}$ are given by

$$
\begin{aligned}
& d_{k}^{\prime}=l_{k}^{\prime-\alpha} 10^{-1.2\left(\theta_{k}^{\prime}-\theta_{\mathrm{tilt}}^{\prime}\right)^{2} / \theta_{3 \mathrm{~dB}}^{\prime 2},} \\
& d_{k}^{\prime \prime}=l_{k}^{\prime \prime-\alpha} 10^{-1.2\left(\theta_{k}^{\prime \prime}-\theta_{\mathrm{tilt}}^{\prime \prime}\right)^{2} / \theta_{3 \mathrm{~dB}}^{\prime \prime 2},}
\end{aligned}
$$

respectively. $l_{k}^{\prime}$ and $\theta_{k}^{\prime}$ are the distance and vertical angle from the $k$ th UE to BS-0. $\theta_{\text {tilt }}^{\prime}$ and $\theta_{3 \mathrm{~dB}}^{\prime}$ are the downtilting angle and HPBW of BS- 0 , respectively. $l_{k}^{\prime \prime}$ and $\theta_{k}^{\prime \prime}$ are the distance and vertical angle from the $k$ th UE to BS-2. $\theta_{\text {tilt }}^{\prime \prime}$ and $\theta_{3 \mathrm{~dB}}^{\prime \prime}$ are the downtilting angle and HPBW of BS-2, respectively. We further assume $\theta_{3 \mathrm{~dB}}=\theta_{3 \mathrm{~dB}}^{\prime}=\theta_{3 \mathrm{~dB}}^{\prime \prime}$ in this paper.

Assuming perfect CSI of active UE in Cell 1 is available at BS-1. As ZF precoding is performed, we have

$$
\mathbf{x}=\mathbf{G}^{\dagger} \text { Ps, }
$$

where $\mathbf{s} \in \mathbb{C}^{K \times 1}$ with $\mathrm{E}\left[\mathbf{s s}^{H}\right]=\mathbf{I}_{K}$ is the information-bearing vector. The $K \times K$ matrix $\mathbf{P}=\operatorname{diag}\left(\sqrt{p_{1}}, \ldots, \sqrt{p_{K}}\right)$ is the power allocating matrix to ensure $\operatorname{tr}\left(\mathbf{x x}^{H}\right) \leq P$; that is,

$$
\operatorname{tr}\left(\mathbf{D}^{-2} \mathbf{P}^{2}\left(\mathbf{H H}^{H}\right)^{-1}\right) \leq P .
$$

And $p_{k}$ is the transmitting power allocated to the $k$ th active $\mathrm{UE}$. Thus, the received signal for the $k$ th UE is

$$
y_{k}=\sqrt{p_{k}} s_{k}+\sqrt{d_{k}^{\prime}} \mathbf{h}_{k}^{\prime} \mathbf{x}^{\prime}+\sqrt{d_{k}^{\prime \prime}} \mathbf{h}_{k}^{\prime \prime} \mathbf{x}^{\prime \prime}+n_{k}
$$

where $s_{k}, n_{k}, \mathbf{h}_{k}^{\prime}$, and $\mathbf{h}_{k}^{\prime \prime}$ are the $k$ th elements of $\mathbf{s}, \mathbf{n}$, the $k$ th row of $\mathbf{H}^{\prime}$, and the $k$ th row of $\mathbf{H}^{\prime \prime}$, respectively. The effective signal-to-interference-plus-noise ratio (SINR) at the $k$ th UE is

$$
\gamma_{k}=\frac{p_{k}}{\sigma_{n}^{2}+d_{k}^{\prime} \mathbf{h}_{k}^{\prime} \mathbf{x}^{\prime} \mathbf{x}^{\prime H} \mathbf{h}_{k}^{\prime H}+d_{k}^{\prime \prime} \mathbf{h}_{k}^{\prime \prime} \mathbf{x}^{\prime \prime} \mathbf{x}^{\prime \prime H} \mathbf{h}_{k}^{\prime \prime H}} .
$$

Therefore, the system sum rate of the $n$th subslot can be calculated as

$$
\mathscr{R}=\sum_{k=1}^{K} \log _{2}\left(1+\gamma_{k}\right)
$$

\section{Low-Complexity User Scheduling with Switched Antenna Tilting ( $K^{*}$-RRS-SAT)}

In this section, the proposed user scheduling scheme, $K^{*}$ RRS-SAT, is discussed in detail. First, we analyze the system sum rate performance in large-system regime with the RMT tools. Then, a low-complexity user scheduling scheme is proposed based on the asymptotic sum rate analysis.

3.1. Asymptotic Sum Rate Analysis. We discuss the asymptotic sum rate performance of the $n$th subslot as $M, K \rightarrow \infty$ with a fixed ratio $K / M>0$. The key point is to obtain the asymptotic value of the SINR given by (10).

In the large-system regime, the SINR of each UE tends to a value independent of the instantaneous SSF CSI that we can obtain by applying the RMT tools. As the entries of $\mathbf{h}_{k}^{\prime}, \mathbf{h}_{k}^{\prime \prime}, \mathbf{x}^{\prime}$, and $\mathbf{x}^{\prime \prime}$ are i.i.d. and $\mathbf{x}^{\prime} \mathbf{x}^{\prime H}, \mathbf{x}^{\prime \prime} \mathbf{x}^{\prime \prime H}$ have uniformly bounded spectral norms, it is easy to see that the conditions of [17, Corollary 1] are satisfied. Hence, we have

$$
\begin{gathered}
\mathbf{h}_{k}^{\prime} \mathbf{x}^{\prime} \mathbf{x}^{\prime H} \mathbf{h}_{k}^{\prime H} \stackrel{\text { a.s. }}{\longrightarrow} \operatorname{tr}\left(\mathbf{x}^{\prime} \mathbf{x}^{\prime H}\right), \\
\mathbf{h}_{k}^{\prime \prime} \mathbf{x}^{\prime \prime} \mathbf{x}^{\prime \prime H} \mathbf{h}_{k}^{\prime \prime H} \stackrel{\text { a.s. }}{\longrightarrow} \operatorname{tr}\left(\mathbf{x}^{\prime \prime} \mathbf{x}^{\prime \prime H}\right) .
\end{gathered}
$$

Thus, (10) can be rewritten as

$$
\gamma_{k} \stackrel{\text { a.s. }}{\longrightarrow} \frac{p_{k}}{\sigma_{n}^{2}+\left(d_{k}^{\prime}+d_{k}^{\prime \prime}\right) P} \text {. }
$$

Using [18, Theorem 2.4], we have

$$
\operatorname{tr}\left(\mathbf{Y} \mathbf{Y}^{H}+z^{-1} \mathbf{I}_{K}\right)^{-1} \stackrel{\text { a.s. }}{\longrightarrow} \operatorname{tr} \Psi,
$$

in which $\mathbf{Y}=\mathbf{D}^{-1} \mathbf{P H}, \boldsymbol{\Psi}=\operatorname{diag}\left(\psi_{1}, \ldots, \psi_{K}\right)$, and $\psi_{k}$ is the unique solution to the following implicit equations:

$$
\begin{array}{ll}
\psi_{k}\left(z^{-1}\right)=\frac{-z}{\left(1+\sum_{j=1}^{M} d_{k}^{-1} p_{k} \widetilde{\psi}_{j}\left(z^{-1}\right)\right)}, \quad 1 \leq k \leq K, \\
\tilde{\psi}_{j}\left(z^{-1}\right)=\frac{-z}{\left(1+\sum_{k=1}^{K} d_{k}^{-1} p_{k} \psi_{k}\left(z^{-1}\right)\right)}, \quad 1 \leq j \leq M .
\end{array}
$$

Substituting (16) into (15), we find

$$
\psi_{k}\left(z^{-1}\right)=\left(-z^{-1}+\frac{M d_{k}^{-1} p_{k}}{1+\sum_{i=1}^{K} d_{i}^{-1} p_{i} \psi_{i}}\right)^{-1} .
$$

Therefore, as a special case of $z \rightarrow+\infty$, (8) can be rewritten as

$$
\sum_{k=1}^{K} d_{k}^{-1} p_{k} \leq P(M-K)
$$

In this paper, we consider the equal power allocation, which is widely used in LSAS. According to (18), the power allocated to each active UE is

$$
p_{1}=\cdots=p_{K}=\frac{P(M-K)}{\sum_{k=1}^{K} d_{k}^{-1}} .
$$

Thus, the sum rate is given by

$$
\mathscr{R}_{\mathrm{eq}} \stackrel{\text { a.s. }}{\longrightarrow} \sum_{k=1}^{K} \log _{2}\left(1+\frac{P(M-K)}{\sum_{k=1}^{K} d_{k}^{-1}\left(\sigma_{n}^{2}+d_{k}^{\prime} P+d_{k}^{\prime \prime} P\right)}\right) \text {. }
$$

Applying Jensen's inequality

$$
\mathrm{E}\left[\log _{2}\left(1+\frac{1}{x}\right)\right] \geq \log _{2}\left(1+\frac{1}{\mathrm{E}[x]}\right)
$$


the lower bound $\overline{\mathscr{R}}$ of the asymptotic ergodic sum rate $\mathscr{R}_{\text {eq }}$ (20) can be calculated as

$$
\overline{\mathscr{R}}=K \log _{2}\left(1+\frac{P(M-K)}{\sigma_{n}^{2} T_{1}+P T_{2}+P T_{3}}\right),
$$

where

$$
\begin{gathered}
T_{1}=\mathrm{E}\left[\sum_{k=1}^{K} d_{k}^{-1}\right], \\
T_{2}=\mathrm{E}\left[\left(d_{k}^{\prime}+d_{k}^{\prime \prime}\right) d_{k}^{-1}\right], \\
T_{3}=\mathrm{E}\left[\sum_{i=1, i \neq k}^{K}\left(d_{k}^{\prime}+d_{k}^{\prime \prime}\right) d_{i}^{-1}\right] .
\end{gathered}
$$

We employ the circle cell model [19] with the single-cell radius $R_{\max }$ in this paper. The points in horizontal plane are denoted by polar coordinates; for example, BS-1 is located at $(0,0), \mathrm{BS}-2$ is at $\left(2 R_{\max }, 0\right)$, and the $k$ th UE is at $\left(r_{k}, \phi_{k}\right)$. Moreover, we assume the BSs have the same height $h_{\mathrm{BS}}$ and all the UE items have the same height $h_{\mathrm{UE}}$. Therefore, we have

$$
\begin{gathered}
l_{k}=\sqrt{r_{k}^{2}+\Delta h^{2}}, \\
l_{k}^{\prime}=\sqrt{r_{k}^{2}+4 R_{\max }^{2}-4 R_{\max } r_{k} \cos \phi_{k}+\Delta h^{2}}, \\
l_{k}^{\prime \prime}=\sqrt{r_{k}^{2}+4 R_{\max }^{2}+4 R_{\max } r_{k} \cos \phi_{k}+\Delta h^{2}}, \\
\theta_{k}^{\prime}=\arctan \left(\frac{\Delta h}{r_{k}}\right), \\
\theta_{k}^{\prime \prime}=\arctan \left(\frac{\Delta h}{\sqrt{r_{k}^{2}+4 R_{\max }^{2}-4 R_{\max } r_{k} \cos \phi_{k}}}\right), \\
\left.\frac{\Delta h}{\sqrt{r_{k}^{2}+4 R_{\max }^{2}+4 R_{\max } r_{k} \cos \phi_{k}}}\right),
\end{gathered}
$$

where $\Delta h=h_{\mathrm{BS}}-h_{\mathrm{UE}}$. Assuming all the candidate UEs are independent uniformly distributed (i.u.d.), as UE is selected in the manner of RRS at each subslot, we attain that all the active UE items served at a subslot are also i.u.d. in the corresponding circular ring region. Let $R_{i}$ and $R_{o}$ indicate the inner and outer radii of the circular ring region, respectively. The probability density functions (PDFs) of $r_{k}$ and $\phi_{k}, k=1, \ldots$, $K$ are given by

$$
\begin{gathered}
f_{R}\left(r_{k}\right)=\frac{2 r_{k}}{R_{o}^{2}-R_{i}^{2}}, \quad R_{i} \leq r_{k} \leq R_{o} \\
f_{\Phi}\left(\phi_{k}\right)=\frac{1}{2 \pi}, \quad 0 \leq \phi_{k}<2 \pi,
\end{gathered}
$$

respectively.
With (25) and (26), we can rewrite (23) as

$$
\begin{aligned}
& T_{1}=K E\left[d_{k}^{-1}\right] \\
& =K \int_{R_{i}}^{R_{o}} \int_{0}^{2 \pi} l_{k}^{\alpha} 10^{1.2\left(\theta_{k}-\theta_{\mathrm{tilt}}\right)^{2} / \theta_{3 \mathrm{~dB}}^{2}} \\
& \cdot f_{R}\left(r_{k}\right) f_{\Phi}(\phi) \mathrm{d} r_{k} \mathrm{~d} \phi_{k}, \\
& T_{2}=\int_{R_{i}}^{R_{o}} \int_{0}^{2 \pi} l_{k}^{\alpha}\left(l_{k}^{\prime-\alpha}+l_{k}^{\prime \prime-\alpha}\right) 10^{1.2\left(\theta_{k}-\theta_{\text {tilt }}\right)^{2} / \theta_{3 \mathrm{~dB}}^{2}} \\
& \cdot 10^{-1.2\left(\theta_{k}^{\prime}-\theta_{\text {tilt }}^{\prime}\right)^{2} / \theta_{3 \mathrm{~dB}}^{2}} \\
& \cdot 10^{-1.2\left(\theta_{k}^{\prime \prime}-\theta_{\text {tilt }}^{\prime \prime}\right)^{2} / \theta_{3 \mathrm{~dB}}^{2}} \\
& \text { - } f_{R}\left(r_{k}\right) f_{\Phi}\left(\phi_{k}\right) \mathrm{d} r_{k} \mathrm{~d} \phi_{k}, \\
& T_{3}=(K-1) \mathrm{E}\left[d_{k}^{\prime}+d_{k}^{\prime \prime}\right] \mathrm{E}\left[d_{k}^{-1}\right] \\
& =\frac{(K-1) T_{1}}{K} \int_{R_{i}}^{R_{o}} \int_{0}^{2 \pi}\left(l_{k}^{\prime-\alpha}+l_{k}^{\prime \prime-\alpha}\right) 10^{-1.2\left(\theta_{k}^{\prime}-\theta_{\mathrm{tilt}}^{\prime}\right)^{2} / \theta_{3 \mathrm{~dB}}^{2}} \\
& \cdot 10^{-1.2\left(\theta_{k}^{\prime \prime}-\theta_{\text {tilt }}^{\prime \prime}\right)^{2} / \theta_{3 \mathrm{~dB}}^{2}} \\
& \text { - } f_{R}\left(r_{k}\right) f_{\Phi}\left(\phi_{k}\right) \mathrm{d} r_{k} \mathrm{~d} \phi_{k}
\end{aligned}
$$

Then, substituting (24) and (27) into (22), we can see that $\overline{\mathscr{R}}$ only depends on system parameters (i.e., $M, K, R_{i}, R_{o}, R_{\max }$, $\Delta h, \theta_{\text {tilt }}, \theta_{\text {tilt }}^{\prime}, \theta_{\text {tilt }}^{\prime \prime}, \theta_{3 \mathrm{~dB}}, P$, and $\left.\sigma_{n}^{2}\right)$. In other words, $\mathscr{\mathscr { R }}$ can be expressed as a function $g(\cdot)$ of the system parameters; that is,

$$
\overline{\mathscr{R}}=g\left(M, K, P, \sigma_{n}^{2}, R_{\max }, R_{i}, R_{o}, \Delta h, \theta_{\text {tilt }}, \theta_{\text {tilt }}^{\prime}, \theta_{\text {tilt }}^{\prime \prime}, \theta_{3 \mathrm{~dB}}\right) .
$$

Obviously, no instantaneous CSI is needed to solve $\overline{\mathscr{R}}$, which implies that we can optimize the variables in (28) offline to maximize $\overline{\mathscr{R}}$.

Remark 1. Though we cannot obtain the closed-form expression of $g(\cdot)$, the value of (28) can be solved easily with MATLAB.

Remark 2. The approximate sum rate $\overline{\mathscr{R}}$ solved by (28) is for the $n$th UE group. If no UE partition (or called cell splitting) is taken (i.e., $N=1$ ), the BS selects active UE among candidate UE items all over the cell. We will have $R_{o}=R_{\max }, R_{i}=$ $R_{\text {min }}$, where $R_{\text {min }}$ is a predefined reference value in the system model, indicating the minimum horizontal distance from UE to BS [19].

3.2. Low-Complexity $K^{*}-R R S-S A T$ Scheme. It is well known that, for AAS, the vertical angles from UE to BS impose a significant impact on the LSF channel gains $[3,4,20]$. If the $\mathrm{UE}$ is far away from the main lobe in vertical directions, the antenna gain will be much smaller than that of the UE within the antenna main lobe range. Accordingly the antenna adjusting plays a critical role in enhancing the performance of AAS. 


\begin{tabular}{|c|l|c|c|c|c|}
\hline BS-1 $\begin{array}{c}K_{1}^{*} \text { UE in } \\
\text { group 1 }\end{array}$ & $\ldots$ & $\begin{array}{c}K_{N}^{*} \text { UE in } \\
\text { group } N\end{array}$ & $\begin{array}{c}K_{1}^{*} \text { UE in } \\
\text { group 1 }\end{array}$ & $\ldots$ & $\begin{array}{c}K_{N}^{*} \text { UE in } \\
\text { group N }\end{array}$ \\
\hline
\end{tabular}

\begin{tabular}{|c|c|c|c|c|c|}
\hline BS-2 \begin{tabular}{c|c|c|c|}
$K_{N}^{*}$ UE in \\
group N
\end{tabular} & $\ldots$ & $\begin{array}{c}K_{1}^{*} \text { UE in } \\
\text { group 1 }\end{array}$ & $\begin{array}{c}K_{N}^{*} \text { UE in } \\
\text { group N }\end{array}$ & $\ldots$ & $\begin{array}{c}K_{1}^{*} \text { UE in } \\
\text { group 1 }\end{array}$ \\
\hline Subslot 1 & Slot 1L & Subslot N & Subslot 1 & Subslot N \\
\hline
\end{tabular}

FIgURE 2: UE scheduling diagram of the proposed scheme. The scheduled group order in Cell 1 is $\{1,2, \ldots, N\}$, and the order in Cell 2 is $\{N, N-1, \ldots, 1\}$.

In addition, for LSAS, the number of simultaneously served UE items is important to ensure good sum rate performance. Particularly, as mentioned in [12], with the equal power allocated ZF precoding, making fully spatial multiplexing of the BS antennas usually does not provide the optimal sum rate performance, which means that, for maximizing the system sum rate performance, the BS should not serve as many UE items as possible at the same time. Hence, the user scheduling needs to be carefully considered for LSAS with a large number of candidate UE items. Furthermore, for practical LSAS, online computational resources have become a bottleneck and thus computational complexity has become one of the most important performance indexes [1]. The tendency towards low-complexity online signal processing is already quite clear for LSAS.

Aiming to achieve good sum rate performance with low computational complexity in LS-AAS, we develop a lowcomplexity UE scheduling method together with switched antenna tilting based on UE partition and BS antenna tilting optimization, referred to as $K^{*}$-RRS-SAT. Figure 2 shows the time slot diagram of the proposed scheduling scheme.

The details of $K^{*}$-RRS-SAT are shown as follows.

Step 1. We divide the whole cell into $N$ circular ring regions. The inner and outer radii $R_{i}$ and $R_{o}$ for the $n$th region, denoted by $R_{n-1}, R_{n}$, are determined according to the vertical HPBW of the BS antennas. UE located in the same ring area is then assigned to one UE group. Each scheduling slot is also divided into $N$ equal-time subslots.

The solution of $N, R_{n-1}$ and $R_{n}$ for the UE partition is shown as follows. The vertical angle range of the whole UE all over the cell area is given by $\left[\Theta_{\min }, \Theta_{\max }\right]$, where

$$
\Theta_{\text {min }}=\arctan \frac{\Delta h}{R_{\max }}, \quad \Theta_{\max }=\arctan \frac{\Delta h}{R_{\min }} .
$$

Therefore, the number of UE groups is calculated as

$$
N=\left\lceil\frac{\Theta_{\max }-\Theta_{\min }}{\theta_{3 \mathrm{~dB}}}\right\rceil,
$$

where $\lceil\cdot\rceil$ denotes the ceiling function. The angle range of each $\mathrm{UE}$ group is then given by

$$
\Delta \Theta=\frac{\left(\Theta_{\max }-\Theta_{\min }\right)}{N} .
$$

The angle bounds of the $n$th $(1 \leq n \leq N)$ UE group are

$$
\begin{gathered}
\Theta_{n-1}=\Theta_{\min }+(n-1) \Delta \Theta, \\
\Theta_{n}=\Theta_{\min }+n \Delta \Theta,
\end{gathered}
$$

respectively. Thus, the bounds of the horizontal distance from UE to BS- 1 for the $n$th UE group are given by

$$
R_{n-1}=\frac{\Delta h}{\tan \Theta_{n-1}}, \quad R_{n}=\frac{\Delta h}{\tan \Theta_{n}} .
$$

Denoting the average number of candidate UE items in the $n$th UE group by $C_{n}$, we have

$$
C_{n}=\operatorname{rnd}\left(C \frac{R_{n}^{2}-R_{n-1}^{2}}{R_{\max }^{2}-R_{\min }^{2}}\right),
$$

where $\operatorname{rnd}(\cdot)$ is the rounding operation.

Step 2. At the $n$th subslot, BS-1 schedules $K_{n}^{*}$ UE from the $n$th UE group in Cell 1 with antenna tilting angle $\theta_{\text {tilt,n }}^{*}$ (i.e., $\left.K_{n}=K_{n}^{*}, \theta_{\text {tilt }, n}=\theta_{\text {tilt, } n}^{*}\right)$. BS-0 and BS-2 schedule $K_{N-n+1}^{*}$ UE from the $(N-n+1)$ th UE groups in Cell 0 and Cell 2 with $\theta_{\text {tilt, } N-n+1}^{*}$, respectively.

In what follows, we discuss how to find $K_{n}^{*}$ and $\theta_{\text {tilt }, n}^{*}$. According to (28), the approximate sum rate at the $n$th subslot is given by

$$
\overline{\mathscr{R}}_{n}=g\left(M, K_{n}, P, \sigma_{n}^{2}, R_{n-1}, R_{n}, \Delta h, \theta_{\text {tilt }, n}, \theta_{\text {tilt }, N-n+1}, \theta_{3 \mathrm{~dB}}\right) .
$$

The average sum rate of a scheduling slot is then calculated as

$$
\overline{\mathscr{R}}_{\text {avg }}=\frac{1}{N} \sum_{n=1}^{N} \overline{\mathscr{R}}_{n} .
$$

Given the value of other variables, $K_{n}^{*}$ and $\theta_{\text {tilt, } n}^{*}(1 \leq n \leq$ $N)$ are the solutions of the following optimization problem (many classical search algorithms [21] can be used to solve the problem.),

$$
\begin{array}{ll}
\max _{\substack{K_{n}, \theta_{\text {tilt, }, n}, 1 \leq n \leq N}} & \overline{\mathscr{R}}_{\text {avg }}, \\
\text { s.t. } & 1 \leq K_{n} \leq \min \left\{C_{n}, M\right\}, \\
& \Theta_{n-1} \leq \theta_{\text {tilt }, n} \leq \Theta_{n} .
\end{array}
$$


TABLE 1: Simulation parameters $[13,14]$.

\begin{tabular}{lc}
\hline Parameter & Value \\
\hline Cell radius: $R_{\max }$ & $500 \mathrm{~m}$ \\
Minimum distance: $R_{\min }$ & $35 \mathrm{~m}$ \\
Large-scale fading model: $l^{\alpha}$ & $10^{-3.53} / l^{3.76}$ \\
Transmission bandwidth: $B$ & $20 \mathrm{MHz}$ \\
Total noise: $B \sigma_{n}^{2}$ & $-96 \mathrm{dBm}$ \\
BS height: $h_{\mathrm{BS}}$ & $32 \mathrm{~m}$ \\
UE height: $h_{\mathrm{UE}}$ & $1.5 \mathrm{~m}$ \\
BS vertical HPBW: $\theta_{3 \mathrm{~dB}}$ & $10^{\circ}$ \\
\hline
\end{tabular}

The constraint $1 \leq K_{n} \leq \min \left\{C_{n}, M\right\}$ in (37) means that the number of active UE items for the $n$th UE group, $K_{n}$, should not be larger than the number of candidate UE items $C_{n}$ and the number of BS antennas $M$. Note that in this paper we assume the number of candidate UE items $C$ is large enough to ensure $C_{n} \geq 1$ for $n=1, \ldots, N$. In the future, we will study the scenarios with small $C$ to improve the universality of our scheme.

It is worth noting that the solutions of (37) are unrelated to the instantaneous CSI of UE items and therefore we can obtain them offline. No extra online resources are occupied. The computational complexity of the proposed $K^{*}$-RRS-SAT is just the same as that of the conventional RRS method.

Remark 3. Without loss of generality, we assume the order of scheduled UE groups at a whole slot in Cell 1 is $\{1,2, \ldots, N\}$. Then, there are $N$ ! different permutations for the scheduled group order in Cell 2, such as $\{N, N-1, \ldots, 1\}$ or $\{2,3, \ldots, N, 1\}$. We just choose $\{N, \ldots, 2,1\}$ in this paper. The best choice of this order for Cell 2 will be investigated in our future work.

\section{Numerical Simulations}

In this section, we demonstrate the sum rate performance of the proposed scheme via Monta Carlo simulations. The simulation parameters are shown in Table 1.

In Figure 3 we verify the accuracy of the approximation (35). The Monta Carlo results are shown by lines and the theoretical results are shown by dots. Besides the parameters included in Table 1 , the transmitting power $P$ is set to be $46 \mathrm{dBm}$. According to (30), the number of UE groups is found to be $N=4$. The BS antenna tilting in the $n$th $(n=1, \ldots, 4)$ UE group is assumed as $\theta_{\text {tilt }, n}=\Theta_{\min }+n \Delta \Theta / 2$, where $\Theta_{\min }$ and $\Delta \Theta$ are defined in Section 3.2. Note that in order to investigate the sum rate performance versus the number of active UE items $K, 1 \leq K \leq M$, we assume the number of candidate UE items $C$ to be quite large (i.e., $C=10^{5}$ ) in this simulation to ensure $C_{n} \geq M$ for each UE group. Subfigures (a), (b), (c), and (d) are for scenarios with different numbers of BS antennas $M=8,16,32$ and 64, respectively. It can be seen that the derived sum rate approximation is accurate even for small $M$. Furthermore, we can observe that the number of active UE items maximizing the sum rate exists and is smaller than $M$.
TABLE 2: $\theta_{\mathrm{tilt}, n}^{*}$ for various UE groups.

\begin{tabular}{cccccc}
\hline & $P(\mathrm{dBm})$ & 40 & 43 & 46 & 49 \\
\hline \multirow{4}{*}{$M=8$} & Group 1 & $35.37^{\circ}$ & $35.37^{\circ}$ & $35.37^{\circ}$ & $35.37^{\circ}$ \\
& Group 2 & $25.59^{\circ}$ & $25.59^{\circ}$ & $25.59^{\circ}$ & $25.59^{\circ}$ \\
& Group 3 & $15.45^{\circ}$ & $15.45^{\circ}$ & $15.45^{\circ}$ & $15.46^{\circ}$ \\
& Group 4 & $4.39^{\circ}$ & $4.45^{\circ}$ & $4.53^{\circ}$ & $4.71^{\circ}$ \\
\hline \multirow{5}{*}{$M=16$} & Group 1 & $35.37^{\circ}$ & $35.37^{\circ}$ & $35.37^{\circ}$ & $35.37^{\circ}$ \\
& Group 2 & $25.59^{\circ}$ & $25.59^{\circ}$ & $25.59^{\circ}$ & $25.59^{\circ}$ \\
& Group 3 & $15.45^{\circ}$ & $15.45^{\circ}$ & $15.45^{\circ}$ & $15.45^{\circ}$ \\
& Group 4 & $4.34^{\circ}$ & $4.37^{\circ}$ & $4.42^{\circ}$ & $4.49^{\circ}$ \\
\hline \multirow{5}{*}{$M=32$} & Group 1 & $35.37^{\circ}$ & $35.37^{\circ}$ & $35.37^{\circ}$ & $35.37^{\circ}$ \\
& Group 2 & $25.59^{\circ}$ & $25.59^{\circ}$ & $25.59^{\circ}$ & $25.59^{\circ}$ \\
& Group 3 & $15.45^{\circ}$ & $15.45^{\circ}$ & $15.45^{\circ}$ & $15.45^{\circ}$ \\
& Group 4 & $4.32^{\circ}$ & $4.33^{\circ}$ & $4.36^{\circ}$ & $4.39^{\circ}$ \\
\hline \multirow{5}{*}{$M=64$} & Group 1 & $35.37^{\circ}$ & $35.37^{\circ}$ & $35.37^{\circ}$ & $35.37^{\circ}$ \\
& Group 2 & $25.59^{\circ}$ & $25.59^{\circ}$ & $25.59^{\circ}$ & $25.59^{\circ}$ \\
& Group 3 & $15.45^{\circ}$ & $15.45^{\circ}$ & $15.45^{\circ}$ & $15.45^{\circ}$ \\
& Group 4 & $4.31^{\circ}$ & $4.31^{\circ}$ & $4.32^{\circ}$ & $4.34^{\circ}$ \\
\hline
\end{tabular}

TABLE 3: $K_{n}^{*}$ for various UE groups.

\begin{tabular}{cccccc}
\hline \multirow{4}{*}{$M=8$} & $P(\mathrm{dBm})$ & 40 & 43 & 46 & 49 \\
\hline \multirow{5}{*}{$M=16$} & Group 1 & 5 & 5 & 5 & 5 \\
& Group 2 & 7 & 7 & 7 & 7 \\
& Group 3 & 7 & 7 & 7 & 7 \\
& Group 4 & 4 & 4 & 5 & 5 \\
\hline \multirow{5}{*}{$M=32$} & Group 1 & 5 & 5 & 5 & 5 \\
& Group 2 & 12 & 12 & 12 & 12 \\
& Group 3 & 15 & 15 & 15 & 15 \\
& Group 4 & 7 & 8 & 9 & 10 \\
\hline \multirow{5}{*}{$M=64$} & Group 1 & 5 & 5 & 5 & 5 \\
& Group 2 & 12 & 12 & 12 & 12 \\
& Group 3 & 31 & 31 & 31 & 31 \\
& Group 4 & 14 & 16 & 18 & 20 \\
\hline & Group 1 & 5 & 5 & 5 & 5 \\
& Group 2 & 12 & 12 & 12 & 12 \\
& Group 3 & 49 & 49 & 49 & 49 \\
& Group 4 & 29 & 33 & 37 & 40 \\
\hline
\end{tabular}

In Figure 4, we compare the average sum rate performance of the proposed $K^{*}$-RRS-SAT scheme with the switched beam tilting scheme in [8] (referred to as SBT) and the optimal tilting 3D cell planning scheme in [5] (referred to as OT). ZF precoders are adopted in all the three schemes to make a fair comparison. The number of candidate UE items is $C=10^{3}$. As part of the proposed scheme, $K_{n}^{*}$ and $\theta_{\text {tilt }, n}^{*}$ for various $P$ obtained from (37) are shown in Tables 2 and 3. Simulation results for $M=8,16,32,64$ are presented in Subfigures (a), (b), (c), and (d), respectively. It can be observed that $K^{*}$-RRS-SAT provides higher average sum rate than the SBT scheme and the OT scheme. This is because both the BS antenna tilting adjustment adaptive to various cell regions and the number of simultaneously served UE items are carefully designed in the proposed scheme in order to 


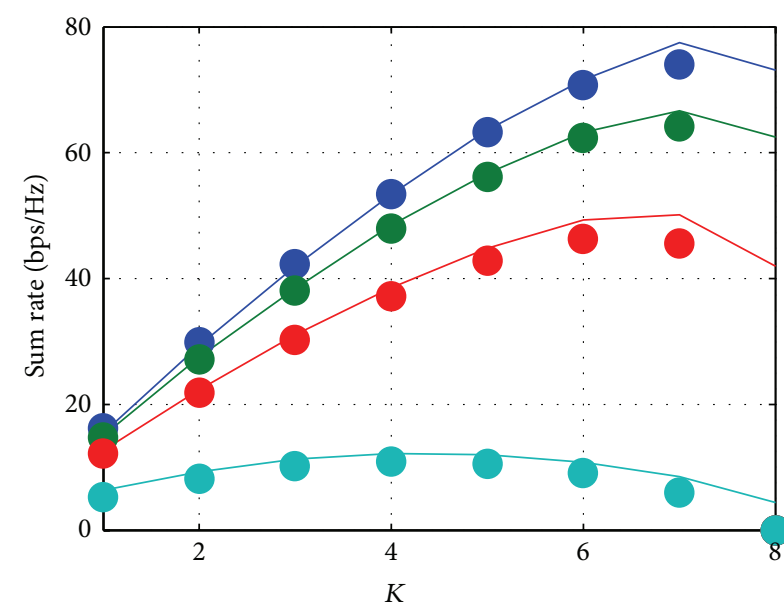

(a) $M=8$

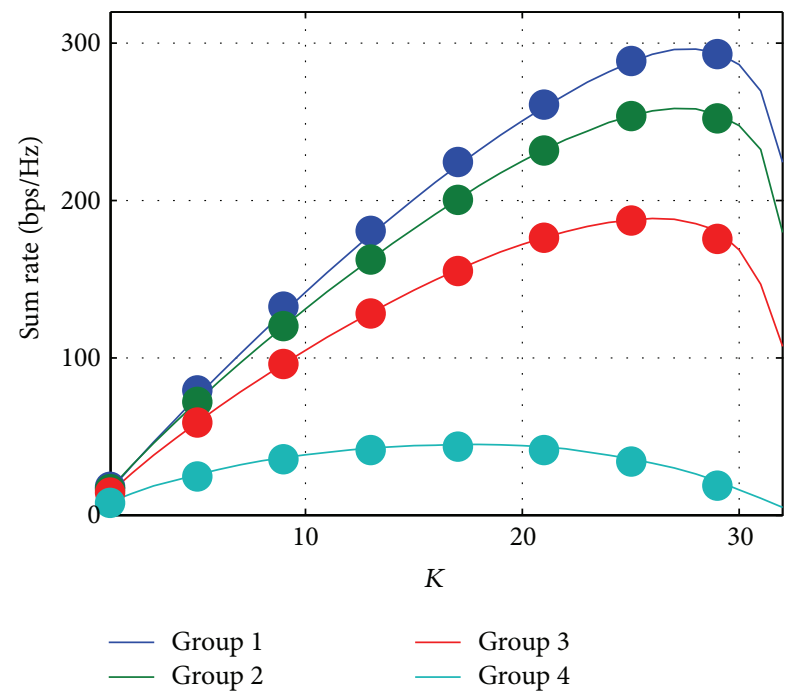

(c) $M=32$

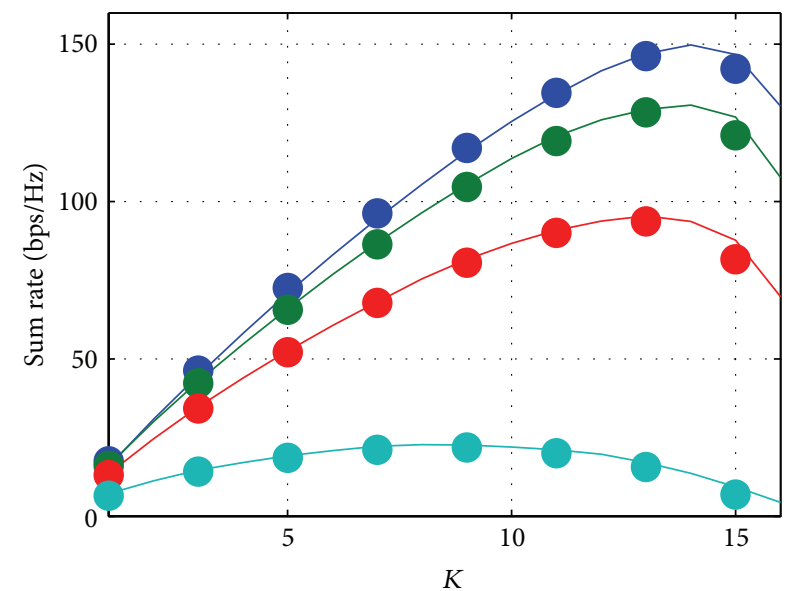

(b) $M=16$

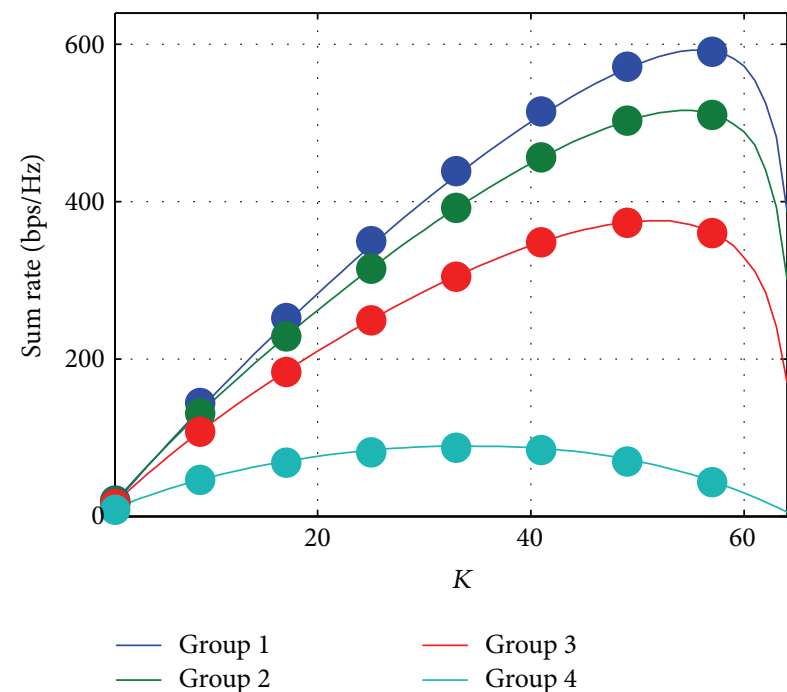

(d) $M=64$

FIgURE 3: Sum rate performance versus number of active UE items K. $P=46 \mathrm{dBm}$.

enhance the average sum rate performance. By contrast, though the cell is divided into several regions in the SBT scheme, the BS antenna tilting angle for each region is just given without optimization (in SBT, each cell is divided into a given number $N$ of equal-area circular ring regions without the consideration of the vertical HPBW of the BS antennas. For Figure 4 , we set $N=4$. The BS antenna tilting angle $\theta_{\text {tilt, } n}$ is given by $\theta_{\text {tilt }, n}=\arctan \left(2 \Delta h /\left(R_{n}+R_{n-1}\right)\right)$, where $R_{n}$ and $R_{n-1}$ are the outer and inner radii of the $n$th circular ring region, respectively. $\Delta h$ represents the difference in height between the BS and the UE. Further, the number of active UE items $K_{n}$ has not been discussed in [8]. To justify the comparison in Figure 4, we select the optimal configuration of $K_{n}$ through Monte Carlo simulations and then plot the average sum rate performance of SBT with the optimal $K_{n}$ ). And in the OT scheme, although the BS antenna tilting has been optimized for maximizing the system sum rate, it is fixed to that value for UE items all over the cell and cannot be adjusted against UE locations (in OT, no cell splitting is employed. The
BS antenna tilting is optimized to serve the entire region of a cell. Moreover, the number of active UE items $K$ has not been studied in [5]. In Figure 4, we choose the optimal value of $K$ for maximizing the OT sum rate and then show the sum rate performance of OT with the optimal $K$ ); hence the potentialities of dynamic adaption of antenna tilting peculiar to AAS are not well exploited. In addition, considering Subfigures (a), (b), (c), and (d) together, we can see that the average sum rates of $K^{*}$-RRS-SAT, SBT, and OT all increase with the number of $\mathrm{BS}$ antennas for the same transmitting power.

\section{Conclusions}

In this paper, we have proposed an effective UE scheduling scheme accompanied with the BS tilting adjusting for multicell downlink LS-AAS. Regarding the feature of AAS, the candidate UE items are divided into several groups according to the HPBW of the BS antenna. Then, exploiting the characteristics of LSAS, the deterministic approximation of the sum 


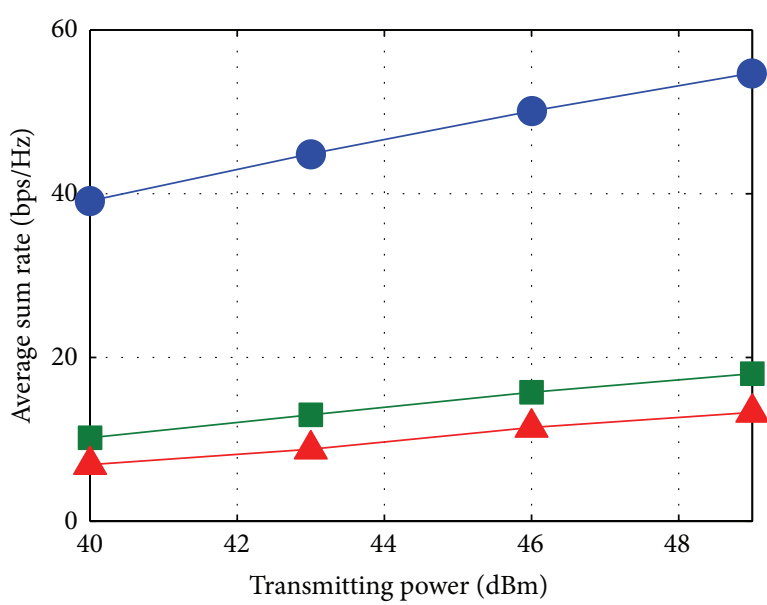

(a) $M=8$

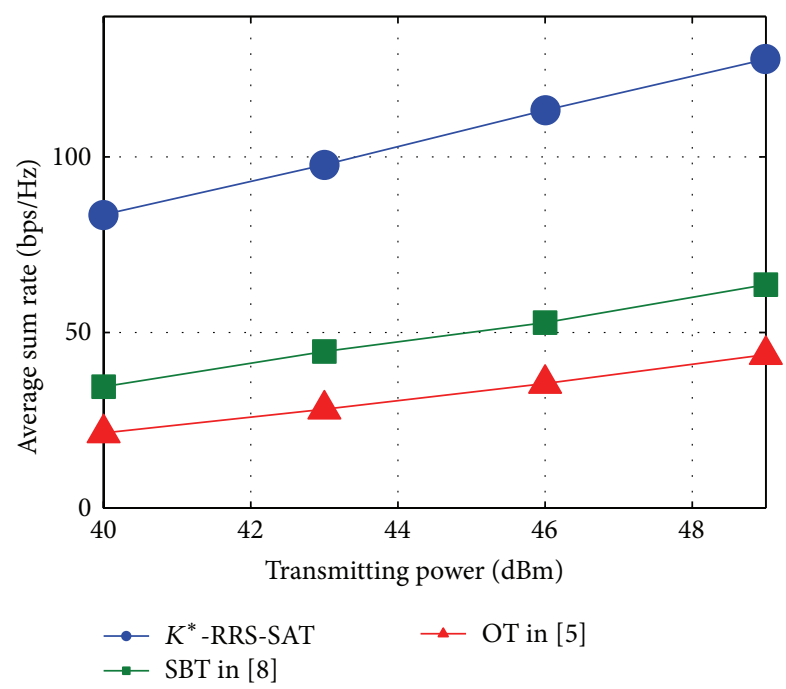

(c) $M=32$

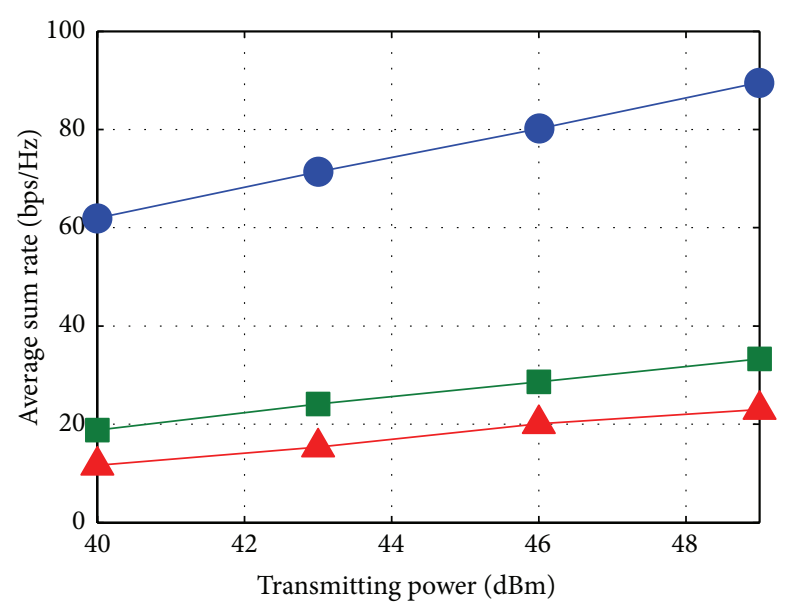

(b) $M=16$

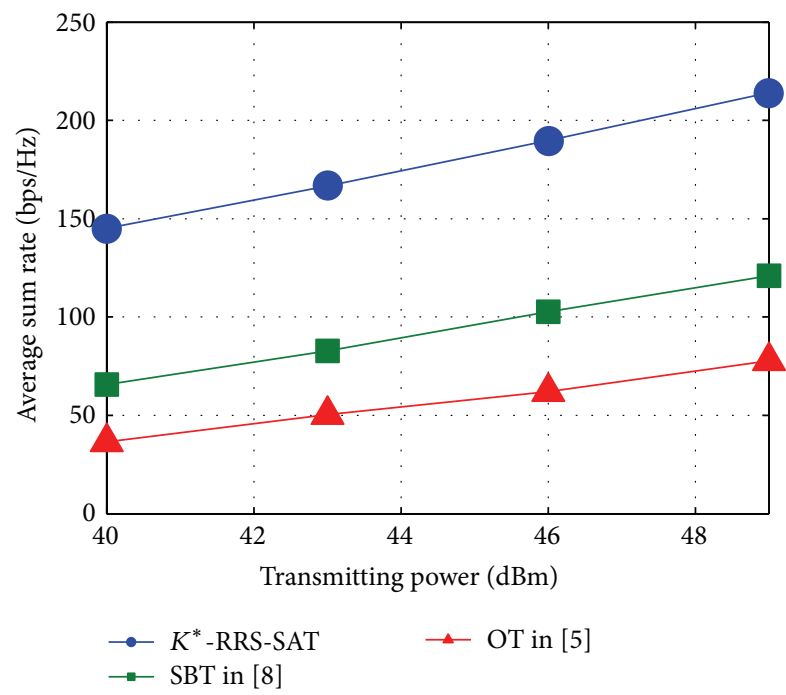

(d) $M=64$

FIGURE 4: Average sum rate performance versus transmitting power $P$ for various schemes.

rate for each UE group is obtained by means of the RMTbased large-system analysis. The optimal number of simultaneously served UE items and the optimal value of BS antenna tilting angle are then solved according to the analytical results to maximize the approximate average sum rate. Moreover, a scheduling slot is also divided into the same number of subslots. At each subslot, the BS adjusts the antenna tilting angle to the optimal value and schedules the optimal number of active UE items in the corresponding UE group for simultaneous data transmission by the RRS method. It is shown that the proposed scheme takes advantage of the features of LSAAS and achieves good sum rate performance with extremely low online computational complexity. In the future, we will consider the $K^{*}$-RRS-SAT scheme in hexagonal 7-cell or 19cell scenarios.

\section{Conflict of Interests}

The authors declared that they have no conflict of interests about this work.

\section{Acknowledgments}

This work was supported by the National Natural Science Foundation of China (NSFC) under Grant nos. 61271188 and 61401041, the Fundamental Research Funds for the Central Universities under Grant no. 2014RC0106, and Beijing Municipal Science and Technology Commission Research Fund Project no. D151100000115002.

\section{References}

[1] F. Rusek, D. Persson, B. K. Lau et al., "Scaling up MIMO: opportunities and challenges with very large arrays," IEEE Signal Processing Magazine, vol. 30, no. 1, pp. 40-60, 2013.

[2] HUAWEI, "Active antenna system: utilizing the full potential of radio sources in the spatial domain," Tech. Rep., HUAWEI, 2012.

[3] H. Halbauer, S. Saur, J. Koppenborg, and C. Hoek, "3D beamforming: performance improvement for cellular networks," Bell Labs Technical Journal, vol. 18, no. 2, pp. 37-56, 2013. 
[4] N. Gresset, H. Halbauer, J. Koppenborg, W. Zirwas, and H. Khanfir, "Interference-avoidance techniques: improving ubiquitous user experience," IEEE Vehicular Technology Magazine, vol. 7, no. 4, pp. 37-45, 2012.

[5] A. Muller, J. Hoydis, R. Couillet, and M. Debbah, "Optimal 3D cell planning: a random matrix approach," in Proceedings of the IEEE Global Communications Conference (GLOBECOM '12), pp. 4512-4517, December 2012.

[6] J. Niemelä, T. Isotalo, and J. Lempiäinen, "Optimum antenna downtilt angles for macrocellular WCDMA network," EURASIP Journal on Wireless Communications and Networking, vol. 2005, no. 5, pp. 816-827, 2005.

[7] N. Seifi, M. Coldrey, and M. Viberg, “Throughput optimization for MISO interference channels via coordinated user-specific tilting," IEEE Communications Letters, vol. 16, no. 8, pp. 12481251, 2012.

[8] N. Seifi, M. Coldrey, and T. Svensson, "Throughput optimization in MU-MIMO systems via exploiting bs antenna tilt," in Proceedings of the IEEE Globecom Workshops (GC Wkshps '12), pp. 653-657, IEEE, Anaheim, Calif, USA, December 2012.

[9] A. Imran, M. A. Imran, A. Abu-Dayya, and R. Tafazolli, "Self organization of tilts in relay enhanced networks: a distributed solution," IEEE Transactions on Wireless Communications, vol. 13, no. 2, pp. 764-779, 2014.

[10] B. Partov, D. J. Leith, and R. Razavi, "Utility fair optimization of antenna tilt angles in LTE networks," IEEE/ACM Transactions on Networking, vol. 23, no. 1, pp. 175-185, 2015.

[11] W. Lee, S.-R. Lee, H.-B. Kong, S. Lee, and I. Lee, "Downlink vertical beamforming designs for active antenna systems," IEEE Transactions on Communications, vol. 62, no. 6, pp. 1897-1907, 2014.

[12] E. Björnson, L. Sanguinetti, J. Hoydis, and M. Debbah, “Optimal design of energy-efficient multi-user MIMO systems: is massive MIMO the answer?," IEEE Transactions on Wireless Communications. In press.

[13] 3GPP TR 36.814, "Further advancements for E-UTRA physical layer aspects (Release 9)," 2010.

[14] 3GPP TR 25.996, Spatial Channel Model for Multiple Input Multiple Output (MIMO) Simulations (Release 9), 2009.

[15] A. D. Wyner, "Shannon-theoretic approach to a Gaussian cellular multiple-access channel," IEEE Transactions on Information Theory, vol. 40, no. 6, pp. 1713-1727, 1994.

[16] W. Lee, S.-R. Lee, H.-B. Kong, and I. Lee, "3D beamforming designs for single user MISO systems," in Proceedings of the IEEE Global Communications Conference (GLOBECOM '13), pp. 3914-3919, Atlanta, Ga, USA, December 2013.

[17] J. Evans and D. N. Tse, "Large system performance of linear multiuser receivers in multipath fading channels," IEEE Transactions on Information Theory, vol. 46, no. 6, pp. 2059-2078, 2000.

[18] W. Hachem, P. Loubaton, and J. Najim, "Deterministic equivalents for certain functionals of large random matrices," The Annals of Applied Probability, vol. 17, no. 3, pp. 875-930, 2007.

[19] K. B. Baltzis, "Hexagonal vs circular cell shape: a comparative analysis and evaluation of the two popular modeling approximations," in Cellular Networks-Positioning, Performance Analysis, Reliability, chapter 4, pp. 103-122, InTech, Rijeka, Croatia, 2011.

[20] N. Seifi, J. Zhang, R. W. Heath, T. Svensson, and M. Coldrey, "Coordinated $3 \mathrm{~d}$ beamforming for interference management in cellular networks," IEEE Transactions on Wireless Communications, vol. 13, no. 10, pp. 5396-5410, 2014.
[21] R. Fletcher, Practical Methods of Optimization, A Wiley-Interscience Publication, Wiley-Interscience, New York, NY, USA, 2nd edition, 1987. 


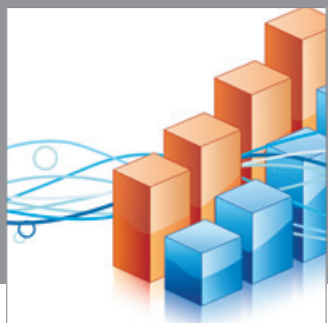

Advances in

Operations Research

mansans

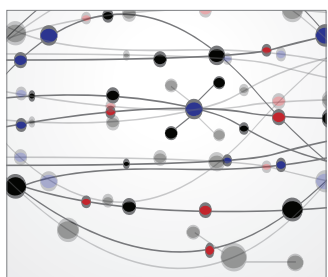

The Scientific World Journal
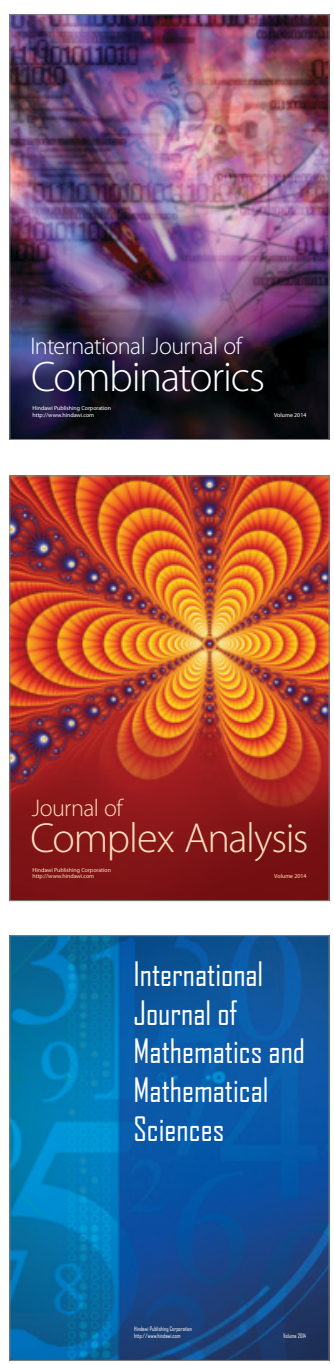
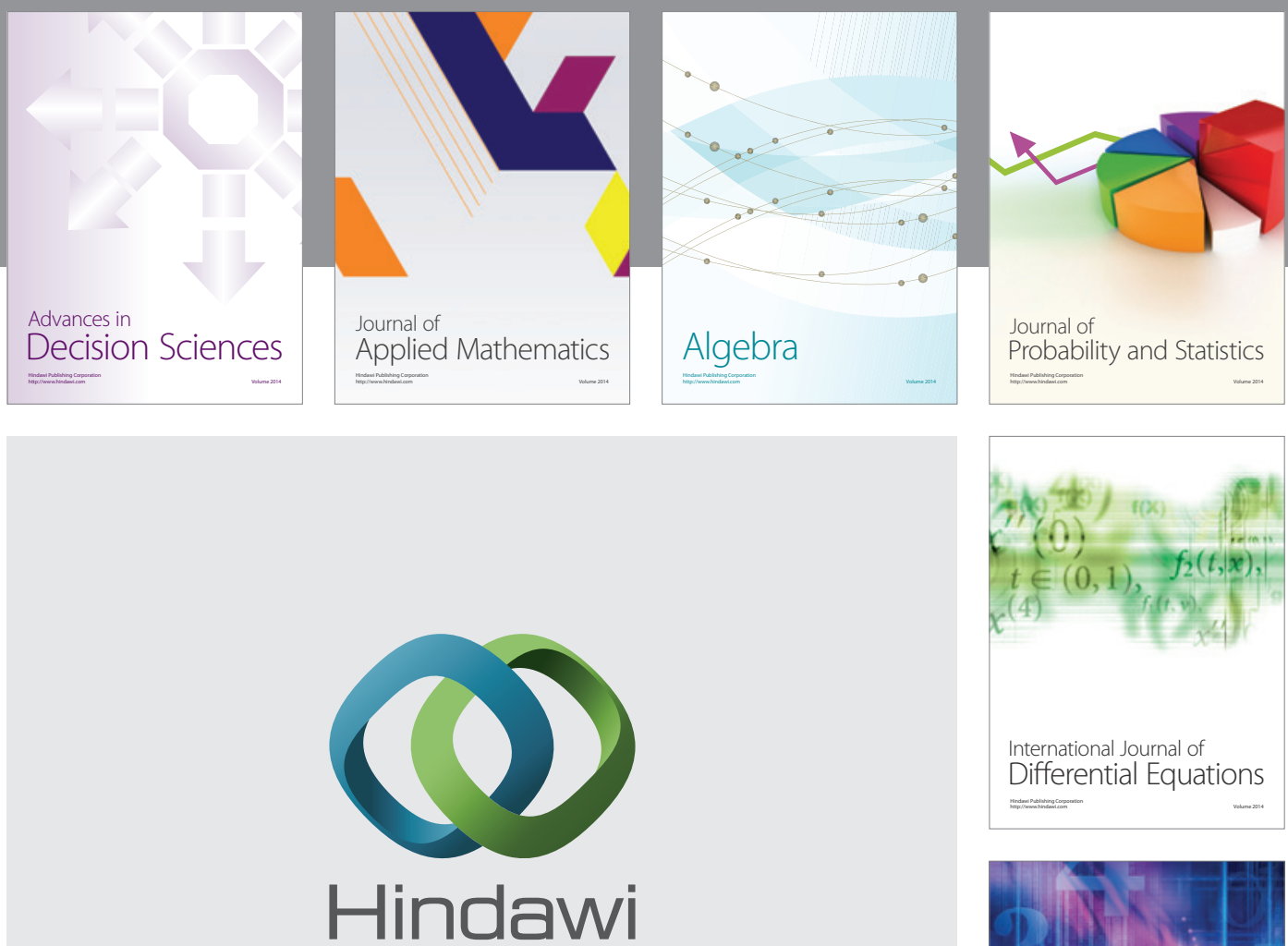

Submit your manuscripts at http://www.hindawi.com
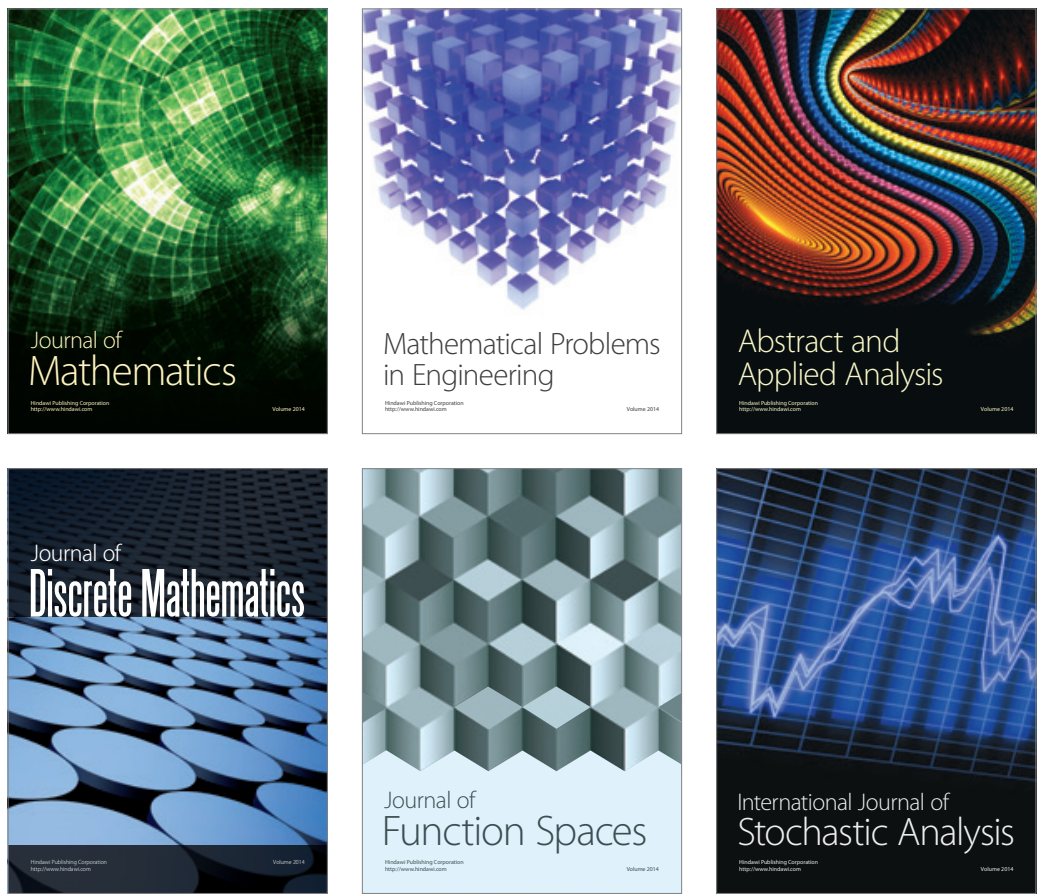

Journal of

Function Spaces

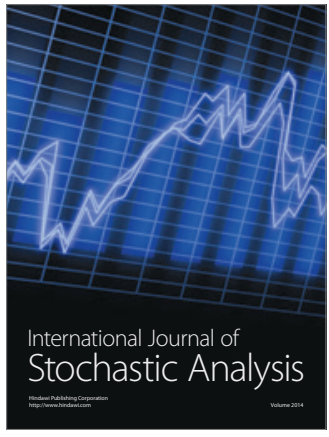

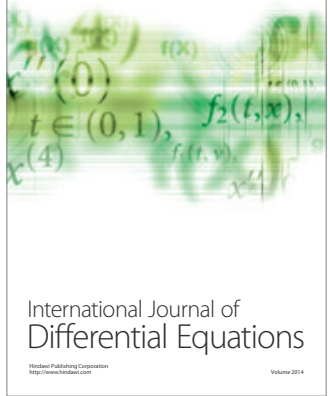
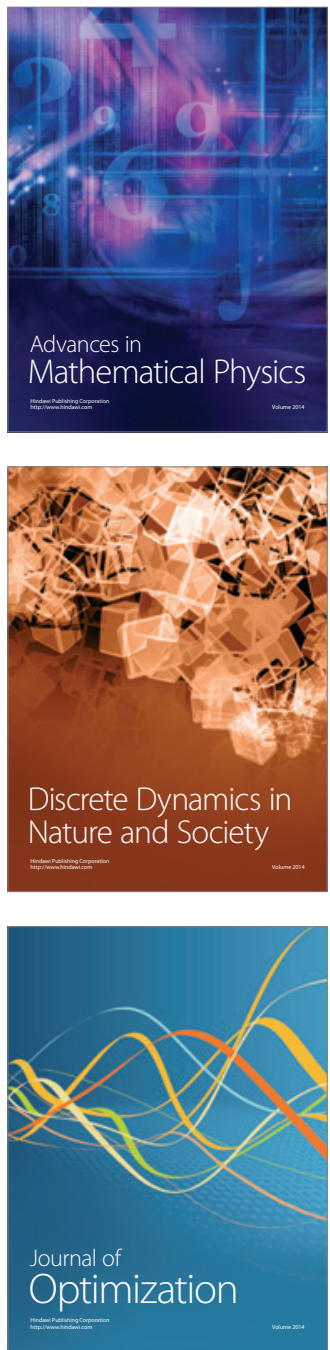